\title{
Assessing Patient Dependence in Alzheimer's Disease
}

\author{
Yaakov Stern, ${ }^{1,2,3}$ Steven M. Albert, ${ }^{1,3}$ Mary Sano, ${ }^{1}$ \\ Marcus Richards, ${ }^{1,3}$ Lisa Miller, ${ }^{1}$ Marshal Folstein, ${ }^{4}$ \\ Marilyn Albert, ${ }^{5}$ Frederick W. Bylsma,${ }^{4}$ and Ginette Lafleche ${ }^{5}$ \\ Departments of ${ }^{1}$ Neurology and ${ }^{2}$ Psychiatry, and the ${ }^{3}$ Sergievsky Center, \\ Columbia University College of Physicians and Surgeons. \\ ${ }^{4}$ Department of Psychiatry and Behavioral Sciences, Johns Hopkins University. \\ spepartments of Psychiatry and Neurology, Massachusetts General Hospital, Harvard Medical School.
}

\begin{abstract}
Background. While cognitive and functional deficits are the hallmark of Alzheimer's disease (AD), loss of social function (and the dependence this implies) is also critical, especially in early stages of disease. Little attention has been directed to this facet of dementing disease. We describe a scale for assessing dependency in $\mathrm{AD}$ and present a baseline profile of dependency in a cohort of AD patients.
\end{abstract}

Methods. In a study of the predictors of the course of AD, 233 patients in early stages of disease (modified MMS $\geqslant 30$ ) were assessed. Psychometric properties of the dependence scale were established. To validate the scale, dependence scores at baseline were correlated with a series of measures assessing cognition and function. The course of dependency over 18 months of follow-up was also analyzed.

Results. The scale shows adequate reliability (test-retest, intraclass correlation). Dependence stage was related to other measures of disease severity. Scalogram analysis shows that the dependence scale is consistent with the course of functional loss established for dementing disease. Prospective data indicate sensitivity of the scale to disease progression.

Conclusion. Dependency is a distinct, measurable component of dementing disease and should be considered an important outcome in studies of $\mathrm{AD}$.

$\mathrm{A}^{\mathrm{L}}$ THOUGH cognitive decline is a hallmark of Alzheimer's disease (AD), a key defining feature of dementia is loss of social or occupational function. When estimating the impact of dementia on a patient and family, it is this aspect of the disease that is often the most disabling. We have developed a new scale for assessing such disability in $\mathrm{AD}$, which we term "dependence."

Several instruments exist for rating functional disability in $\mathrm{AD}$, typically focusing on the ability to perform self-care (e.g., grooming) and instrumental (e.g., taking medications) tasks $(1,2)$. However, these approaches do not fully assess the impact of functional disability on the patient and family. For example, while the Blessed Dementia Rating Scale (BDRS) assesses functional capacity in $\mathrm{AD}$, it does not assess the actual level of care required by the patient. A scale that directly measures such dependence would be valuable, especially in the case of needs not addressed by the BDRS (e.g. , the need to be watched or accompanied when outside). Dependence on others, rather than functional status, is likely to be a better predictor of the service needs of the AD patient, such as the amount of caregiver time or types of support required by a patient.

The need for a scale measuring this aspect of patients' functional decline became evident to us in another context as well. We are in the process of developing a predictor model for the progression of $\mathrm{AD}(3)$. One major milestone in the progression of dementia is admission to a skilled care facility. However, it is well known that many factors influence whether and when a demented patient reaches this endpoint.
Besides the severity of dementia, the decision to admit a patient to a nursing home is influenced by a range of nonmedical factors, including financial, cultural, and familial considerations. In some cases a family may elect to provide all the services required for a patient in the home. For other patients, this help is not available; caregivers are unwilling or unable to provide it, and the result is institutionalization at comparable or even higher levels of function. Thus, institutional placement is not always a good proxy for an elder's level of dependence or need for care.

Here we describe the development, reliability, and validity of a proposed dependence scale. We suggest that a specific level of dependence as rated on this standardized scale can serve as a consistent milestone in the progression of dementia.

\section{METHODS}

\section{Measures}

The dependence scale. - The Dependence Scale is presented in Chart 1. It was adapted from an instrument designed by Gurland (4) for use with community elders. Items deemed to be relevant to the progression of dementia were retained, and scoring was simplified in order to allow administration with minimal training. Items were selected in the hope of assessing as broad a range of dependency as possible. For example, scale items assess relatively subtle types 


\section{Chart 1. Dependence Scale}

A. Does the patient need reminders or advice to manage chores, do shopping, cooking, play games, or handle money?

B. Does the patient need help to remember important things such as appointments, recent events, or names of family or friends?

C. Does the patient need frequent (at least once a month) help finding misplaced objects, keeping appointments, or maintaining health or safety (locking doors, taking medication)?

D. Does the patient need household chores done for them?

E. Does the patient need to be watched or kept company when awake?

F. Does the patient need to be escorted when outside?

G. Does the patient need to be accompanied when bathing or eating?

$H$. Does the patient have to be dressed, washed and groomed?

I. Does the patient have to be taken to the toilet regularly to avoid incontinence?

J. Does the patient have to be fed?

K. Does the patient need to be turned, moved or transferred?

L. Does the patient wear a diaper or a catheter?

M. Does the patient need to be tube fed?

Note. Items $A$ and $B$ are coded: No, 0 ; Occasionally (at least once a month), 1; Frequently (at least once a week), 2 . The remaining items are coded: No, 0 ; Yes, 1.

of dependency, such as the need for reminders and cueing in daily activities (items A-C), as well as more gross forms of dependency (e.g., need for assistance in self-care activities).

All items deal with reported needs of the patient. In some cases the need is simply for supervision, with no specific task linked to this need; other items deal with specific tasks or activities that an elder is unable to perform. This feature of the scale distinguishes it from functional assessment instruments, which stress an elder's capacity to perform a task (or difficulty in performing it) $(5,6)$. The stress on reported needs of the patient also distinguishes the scale from caregiving burden measures, which stress caregiver involvement and the impact of elder support needs on caregivers $(7,8)$.

The scale is designed to be administered to an informant who lives with the patient or is well informed about the patient's day-to-day activities. After the items are administered, the patient's dependence level is derived according to the criteria summarized in Chart 2. These six dependence levels, ranging from no dependence to complete dependence in self-care activities, represented our intuitions about levels of need in an Alzheimer's population. The analysis presented here was conducted in part to assess this scoring convention. Similarly, we wished to explore the usefulness of distinguishing the needs of patients in the case of relatively complex tasks. For this reason, we used a 3-point response format for items $\mathrm{A}-\mathrm{C}$ (no need, occasional need, frequent need).

As an adjunct to the information collected in the depen-
Chart 2. Derivation of Dependence Level

Level $0 \quad(0$ to all items) $\ldots \ldots \ldots \ldots \ldots \ldots \ldots \ldots \ldots$

Level 1 (Either $\mathrm{A}, \mathrm{B}$ or $\mathrm{C}=1) \ldots \ldots \ldots \ldots \ldots \ldots$

Level 2 ( 2 of $A, B$ or $C=1$ or $A$ or $B=2$ or $D=1) \ldots 2$

Level $3(E, F$ or $G=1) \ldots \ldots \ldots \ldots \ldots \ldots \ldots$

Level $4 \quad(H, J$ or $J=1) \ldots \ldots \ldots \ldots \ldots \ldots \ldots$

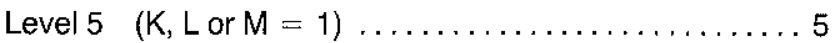

Note. See Chart 1 for dependence scale items.

\section{Chart 3. Equivalent Institutional Care}

Based on the interview and your knowledge of the care the patient received and requires, choose one of the following. Note particularly patients living at home who are receiving the equivalent of institutional care. If the patient is actually living in an institution, choose the level in agreement with current placement.

Limited home care. This includes independent living, with some help in the case of shopping, cooking, or housekeeping, but not with all the tasks $\ldots \ldots \ldots \ldots \ldots \ldots \ldots \ldots \ldots 1$

Adult home. This includes living in a supervised setting (including family members as supervisors) involving regular help with shopping, cooking, and housekeeping; and constant companionship, security, legal, or financial help ....... Health-related facility. This includes around-the-clock supervision of personal care, safety, or medical care ........ 3

dence scale, the interviewer also completes an item that rates the "equivalent institutional care" (EIC) that the patient is receiving. This is simply the interviewer's impression, based on all available information, of the level of care the patient is receiving, regardless of the patient's location. EIC scoring conventions are shown in Chart 3.

Criterion measures for assessing the dependency scale. - In addition to measures of dependency and equivalent institutional care, the following assessments were obtained: Intellectual function was examined using the modified version of the Mini-Mental State Examination (mMMS) (9). This modification of the Folstein Mini-Mental State Examination has a maximum of 57 points, with lower scores indicating poorer cognitive function. Reliability and validity of the scale have been established (10).

Functional capacity was rated using the Blessed Dementia Rating Scale (BDRS) (1). We have reported the results of a factor analysis of this scale, which yielded four well-defined factors: cognitive impairment, self-care needs, personality disturbance, and apathy (2). For this analysis, we wished to know if the dependency measure accounts for variation in outcomes independently of BDRS self-care scores. This would indicate that dependency and self-care capacity represent related but perhaps distinct components of disability in AD.

The Clinical Dementia Rating scale (CDR) (11) was used 
to evaluate the patient at each measurement point to provide a global assessment of the severity of dementia.

Finally, indicators of the patient's current status were recorded. These include work and marital status, living situation (e.g., at home, in nursing home), or death (and cause of death). Characteristics of informants reporting on patients were also noted. These included the relationship of informants to patients, whether informant and patient coresided, and amount of daily contact.

\section{Procedures}

Subjects. - A total of 249 informants representing patients with probable $\mathrm{AD}(12,13)$ were interviewed at baseline. At entry into the Predictors study, patients were required to be at a relatively mild stage of dementia, which was operationalized as a modified mini-mental (mMMS) score $\geqslant$ 30 . Of the 249 patients, 233 had mMMS scores $\geqslant 30$; only these patients are included here. All evaluations were completed as part of the Predictors study described above, whose inclusion/exclusion criteria and procedures have been fully described elsewhere $(14,15)$. Patients were recruited at 3 sites: 104 at Columbia University, 84 at Johns Hopkins University, and 61 at Massachusetts General Hospital. All patients met DSM-II-R criteria for dementia as well as NINCDS-ADRDA criteria for probable AD. Other potential causes of dementia were ruled out using standard diagnostic procedures.

Prospective evaluation. - In the Predictors study, all patients are evaluated every 6 months. To date, 150 patients have been followed for 18 months or more.

\section{Analyses}

Reliability. - In order to assess interrater reliability of the dependence scale, 20 informants were separately interviewed by two different investigators. Intraclass correlations were calculated to assess agreement between raters. Internal consistency of the dependence items was calculated using factor analyses to explore scale dimensionality. Cronbach's alpha was calculated for the scale as a whole and for distinct subsets of items suggested by the factor analysis.

Validity. - Validity of the dependency scale was assessed in a series of three different analyses. First, dependency should correlate cross-sectionally with other indicators of impairment, such as cognitive and functional impairment.

Second, given that the scale assesses dependency in progressive dementing disease, scale items should show a hierarchical or ordinal pattern, such that items indicating greater dependency (e.g., self-care needs) should not occur in the absence of items indicating less gross forms of dependency. This property of the scale was assessed using a Guttman scalogram $(16,17)$.

Finally, with increasing duration of dementing disease, dependency should also increase. Repeated measures analyses of dependency at successive intervals should show an increase in the level of dependency among respondents.
Increases in dependency across the course of disease, controlling for decreases in cognitive or functional performance, would indicate a more general or diffuse impact of dementia on dependency.

RESULTS

Demographic characteristics of the patient sample at baseline are summarized in Table 1. Sixty percent of the sample is female, and the majority of patients are between 70 and 80 years of age. The sample is largely White $(90 \%)$, with the majority $(85 \%)$ born in the U.S. About $45 \%$ of the patients

Table 1. Sample Characteristics at Baseline

\begin{tabular}{|c|c|c|}
\hline & $n$ & $(\%)$ \\
\hline \multicolumn{3}{|l|}{ Gender } \\
\hline Female & 139 & $(60)$ \\
\hline Male & 93 & (40) \\
\hline \multicolumn{3}{|l|}{ Age } \\
\hline$<70$ & 8.5 & (37) \\
\hline $70-80$ & 102 & (44) \\
\hline $80-90$ & 38 & (16) \\
\hline$>90$ & 7 & (3) \\
\hline \multicolumn{3}{|l|}{ Education (yrs) } \\
\hline$<9$ & 33 & (14) \\
\hline $10-12$ & 95 & (41) \\
\hline $13-16$ & 60 & (26) \\
\hline$>16$ & 43 & (19) \\
\hline \multicolumn{3}{|l|}{ Race/Ethnicity } \\
\hline White & 209 & $(90)$ \\
\hline African American & 14 & (6) \\
\hline Latino & 8 & (3) \\
\hline \multicolumn{3}{|l|}{ Natality } \\
\hline U.S. & 210 & $(85)$ \\
\hline \multicolumn{3}{|l|}{ Residential status } \\
\hline Living in community & 212 & (91) \\
\hline Retirement home & 3 & (1) \\
\hline Nursing home & 16 & (8) \\
\hline Other & 2 & (1) \\
\hline \multicolumn{3}{|l|}{ Living arrangement } \\
\hline Living alone & 38 & (16) \\
\hline With spouse & 138 & (59) \\
\hline With children & 12 & (5) \\
\hline With other family/friends & 15 & (6) \\
\hline Other & 16 & (6) \\
\hline Unknown & 17 & (7) \\
\hline \multicolumn{3}{|c|}{ Clinical Dementia Rating Score } \\
\hline Questionable dementia & 9 & (4) \\
\hline Mild & 201 & (86) \\
\hline Moderate & 23 & $(10)$ \\
\hline \multicolumn{3}{|l|}{ Dependency level } \\
\hline 0 & 8 & (3) \\
\hline 1 & 9 & (4) \\
\hline 2 & 149 & (64) \\
\hline 3 & 56 & (24) \\
\hline 4 & 9 & (5) \\
\hline 5 & 2 & (1) \\
\hline \multicolumn{3}{|l|}{ Equivalent Institutional Care } \\
\hline Limited home care & 131 & (56) \\
\hline Adult home & 82 & (35) \\
\hline Health-related facility & 20 & (9) \\
\hline
\end{tabular}


have had more than 12 years of education. At baseline, most patients were residing in the community $(91 \%)$ and living with spouses $(59 \%)$.

As shown in Table 1, CDR levels at baseline did not exceed 2, "moderate dementia"; the majority of patients were scored 1 , "mild dementia" $(86 \%)$. This is consistent with study exclusion criteria (i.e., modified mini-mental score $\geqslant 30$ )

The modal dependence level at baseline was 2 (64\% of patients); level 3 accounted for the majority of the remaining patients $(24 \%)$. In the case of equivalent institutional care (EIC), the majority of patients ( $56 \%$ ) were rated as requiring limited home care.

Informants who reported on patient levels of dependency mostly co-resided with patients $(61 \%)$. Informants were, for the most part, spouses $(57 \%)$ or adult children (32\%). Friends, home health aides, and other family members accounted for the remainder. The majority of informants $(63 \%)$ were in daily contact with subjects; only $10 \%$ had contact less than once a week. While measures of actual caregiving involvement by these informants were not obtained, it would appear that informants were, on the whole, family caregivers with primary responsibility for the welfare of patients.

\section{Scale Reliability}

Interrater reliability. - As described above, 20 informants were independently interviewed by two raters. There was $100 \%$ agreement between the two raters for dependence level. Item scores for the dependency scale were summed, and totals across raters were compared using the intraclass correlation (ICC). The ICC was 0.90 , signifying excellent interrater reliability. Interrater reliability for ratings of equivalent institutional care (EIC) were also examined; the ICC was 0.73 , indicating good reliability.

Internal consistency. - Internal consistency or reliability was calculated using Cronbach's alpha. Again, because all patients had relatively mild dementia severity, relatively few scored positively on the later items. Still, the alpha value was 0.66 , indicating acceptable internal consistency for the scale as a whole.

Examining the internal structure of the scale shows that the 0.66 alpha is a lower bound on the scale's internal consistency. Subjecting responses to principal components analysis (with varimax rotation) shows three clearly defined subscales. The pattern of item loadings (drawn from the sample at 18 months to increase variability in the items tapping greater dependence) is presented in Table 2. Inspection of item loadings shows that the subscales capture a cognitive support dimension (alpha, 0.93), types of assistance in which the elder is active (alpha, 0.87), and types of assistance in which the elder is completely passive (alpha, 0.78).

\section{Scale Validity}

Relationship between dependency and disease severity. - In order to determine if the dependence scale is sensitive to disease severity, we examined cross-sectional relation- ships between dependence level and a series of severity indices, including Clinical Dementia Rating Score (CDR), modified Mini-Mental State Examination (mMMS), Blessed Dementia Rating Scale (BDRS), and Equivalent Institutional Care (EIC). Correlations between dependency level and severity indices are shown in Table 3 . There is a significant correlation between dependence and the mMMS $(r=-.27)$, indicating increased dependence with lower cognitive performance. The correlation between dependency and CDR score was also significant $(r=.34)$, supporting the relationship between poorer cognitive function and increased dependency. The dependence score is highly correlated with two of the BDRS factors, the cognitive $(r=.38)$ and basic self-care $(r=.26)$ factors, while correlations with the two personality change factors were relatively weak. As expected, the correlation with Equivalent Institutional Care was also strong $(r=.58)$, indicating an association between higher levels of dependency and greater need for care.

Since dependence level and the BDRS self-care factor both tap functional deficits in $\mathrm{AD}$ and are correlated, we wished to know if the two were independently related to disease severity. To assess this relationship, we regressed mini-mental (mMMS) scores on the dependence and BDRS self-care measures. Together, the two measures accounted for $15 \%$ of the variance in mMMS scores $\left(R^{2}=.40\right)$, and

Table 2. Factor Loadings for Dependency Items

\begin{tabular}{|c|c|c|c|}
\hline & $\begin{array}{c}\text { Cognitive } \\
\text { Support }\end{array}$ & $\begin{array}{l}\text { Assistance: } \\
\text { Elder Active }\end{array}$ & $\begin{array}{l}\text { Assistance: } \\
\text { Elder Passive }\end{array}$ \\
\hline A. Needs reminding & .95 & -.18 & .08 \\
\hline B. Memory problems & .95 & -.23 & .08 \\
\hline C. Needs guidance & .95 & -.23 & .08 \\
\hline D. Needs chore help & .82 & .15 & .07 \\
\hline E. Must be watched & -.28 & .78 & .02 \\
\hline F. Must escont uutside & -.46 & .67 & .05 \\
\hline G. Must accompany & -.15 & .85 & .08 \\
\hline H. Needs ADL help & .01 & .84 & .15 \\
\hline I. Needs toileting & .04 & .76 & .28 \\
\hline J. Needs to be fed & .13 & .25 & .82 \\
\hline K. Can't move on own & .06 & .08 & .90 \\
\hline L. Must be diapered & .18 & .48 & .58 \\
\hline M. Feeds on tube & .00 & .01 & .82 \\
\hline $\begin{array}{l}\text { Factor alpha } \\
\text { (Cronbach) }\end{array}$ & .93 & .87 & .78 \\
\hline
\end{tabular}

Note. Principal components analysis, varimax rotation.

Table 3. Correlates of Dependency at Baseline

\begin{tabular}{lrcc}
\hline & $r$ & $p$-value & $N$ \\
\hline Modified Mini-Mental & -.27 & .000 & 233 \\
Clinical Dementia Rating score & .34 & .000 & 233 \\
BDRS-Cognition & .38 & .000 & 191 \\
BDRS-Personality & .08 & .147 & 191 \\
BDRS-Apathy & .17 & .008 & 191 \\
BDRS-Self-Care & .26 & .000 & 191 \\
Equivalent Institutional Care rating & .58 & .000 & 233 \\
\hline
\end{tabular}

Note. BDRS factors calculated according to (2), 
each accounted for unique and significant proportions of the variance $(p<.01$ and $p<.001$, respectively).

We also investigated the relationship between dependence level at baseline and patients' living situations. Patients were divided into 3 groups: living alone $(n=38)$, living at home with family or other caregivers $(n=165)$, and living in a health-related facility $(n=16)$. The relationship between dependence level and living situation was highly significant $(p<.001)$. In a logistic regression model with dependency level and BDRS self-care score as predictors, living situation (living alone vs living with others) was strongly associated with dependency level $(p<.001)$; BDRS self-care scores did not independently account for variance in living situation.

Hierarchical structure of scale items. - Table 4 shows the dependency profile of the sample at baseline in the form of Guttman scale. The Guttman scale assesses the degree to which the items are cumulative and hierarchical, that is, how well the items form a scale indicating progressively greater dependency. Respondents dependent in the more extreme indicators of dependency should also be dependent on all items indicating less extreme forms of dependence.

The dependency scale items meet the requirement of hierarchical ordering, as indicated by a coefficient of reproducibility above 0.90 . The baseline scalogram also exceeds 0.60 for its coefficient of scalability, a second indicator of the cumulative nature of dependency assessed by the scale items. Inspection of the dependency scalogram shows that at baseline no respondents were dependent in three of the most severe indicators of dependency, i.e., the need for tubefeeding, need for help in transferring, and the inability to eat independently. The most severe dependency type at baseline is diapering, and requiring this sort of support predicts that respondents will be dependent in all the other items, in the order shown in Table 4. The majority of respondents at baseline do not show this level of dependency, but rather dependency in cognitive items only. For example, 52 respondents need help in completing household chores, need to be reminded about the names of family members, need guidance in finding objects and taking medications, and have significant memory problems; 95 show dependency in the latter three items but no dependency in completing household chores.

\section{Dependence at Follow-Up}

Data for 150 patients were available at four time points, yielding 18 months of follow-up for this subsample of patients. The distribution of dependence and equivalent institutional care stages over this prospective period is shown in Table 5. Mean dependence level at baseline was $2.24( \pm .77)$; at 6 months $2.50( \pm .74)$; at 12 months 2.69 $( \pm .81)$, and at 18 months 2.94 ( \pm .99$)$, showing an increasing trend. EIC level showed a similar increase over the follow-up period: $1.52( \pm .65)$ at baseline; $1.75( \pm .70)$ at 6 months; $1.93( \pm .72)$ at 12 months; and $2.09( \pm .72)$ at 18 months.

These data were subjected to a repeated measures MANOVA with testing interval as the main effect, along with univariate tests for linear time trends. In addition, the same analysis was performed on the dependency scale subfactors, described earlier, to see which component of dependency was responsible for observed time-dependent effects.

Table 4. Baseline Dependency Profile

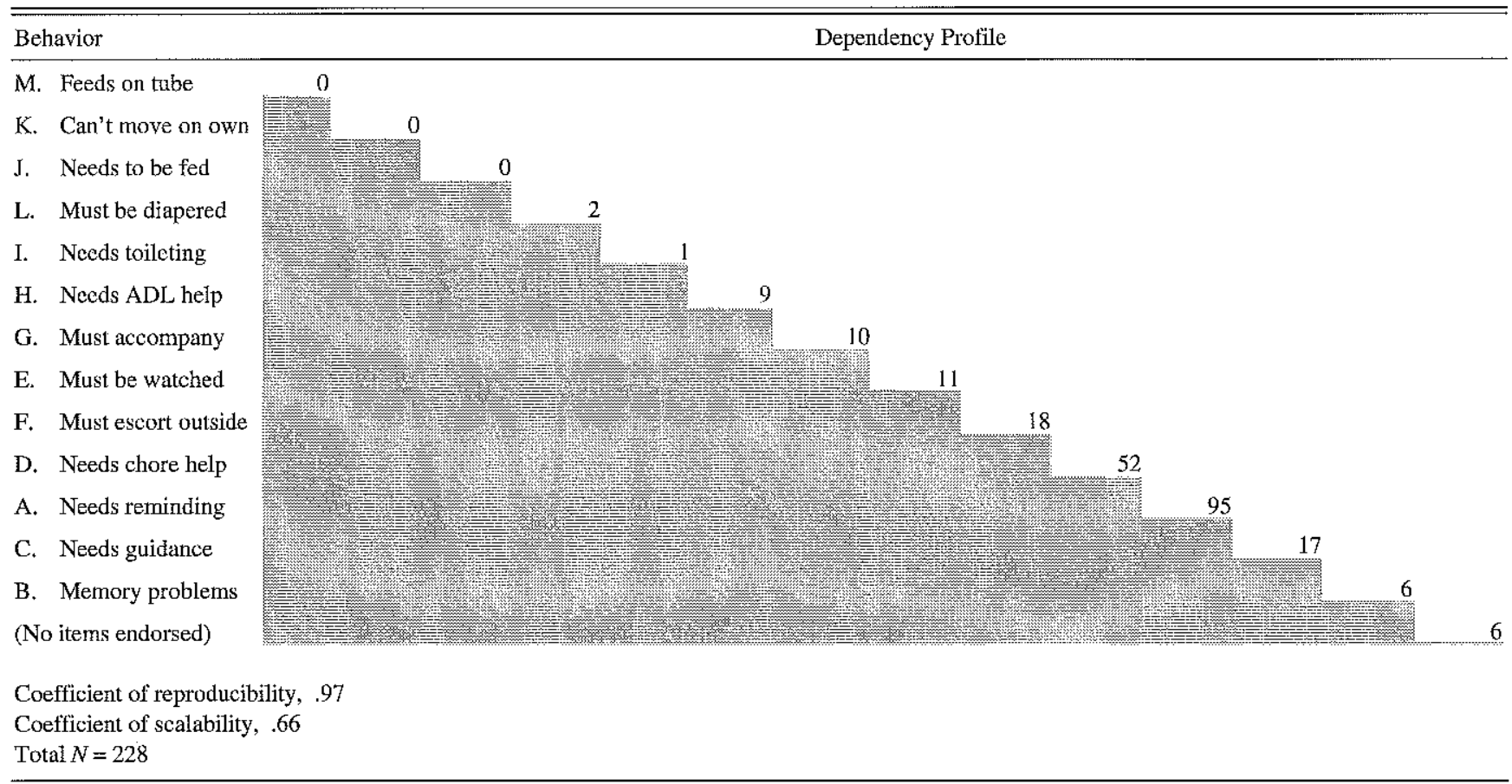

Note. Step-like order of behaviors indicates relative "difficulty" of an item; respondents dependent in an item are likely to be dependent in all behaviors beneath it. Each column represents a dependency profile shown by number of respondents on line. Reproducibility $=(1-$ total errors/total number of responses $)$. Scalability $=($ Reproducibility - marginal errors $) /(1-$ marginal errors $)$. 
Table 5. Mean (SD) Dependency and Care Levels

\begin{tabular}{lcccc}
\hline \hline & Baseline & 6 Months & 12 Months & 18 Months \\
\hline Dependency level & $2.24(.77)$ & $2.50(.75)$ & $2.69(.81)$ & $2.94(.99)$ \\
Equivalent Institutional Care & $1.52(.65)$ & $1.75(.70)$ & $1.93(.72)$ & $2.09(.72)$ \\
$\quad N$ & 233 & 191 & 175 & 140 \\
\hline
\end{tabular}

Note. Dependency range, 0-5, higher score greater dependency; Equivalent Institutional Care range, $1-3(1=$ Limited home care; $2=$ Adult home; $3=$ Health-related facility).

Multivariate effects were significant for dependency (Hotelling's $F=29.68, p<.001)$, for EIC $(F=26.95, p<$ .001 ), and for two of the three dependency subscales (assistance, with elder active, $F=30.28, p<.001$; assistance, with elder passive, $F=3.58, p<.02$ ). The cognitive support subscale did not show this time-related increase in dependency $(F=2.38, p<.08)$, perhaps because the patient sample, on the whole, entered the study with such high levels of dependency in this domain.

To further explore longitudinal trends in dependency, the follow-up data were reanalyzed, this time with BDRS cognitive and self-care scores treated as time-dependent covariates. While such adjustment lowers the multivariate $F$ ratio, the time trend for increased dependency remains significant.

\section{DisCUSSION}

The dependence scale was designed to assess levels of need, and hence care required, as a result of deficits typical of AD. Preliminary evidence suggests that the scale has excellent reliability and validity.

The validity of the scale was addressed in three ways. First, in the baseline data, dependence stage was related to other indices of disease severity, such as degree of cognitive deficit (mMMS, CDR) and functional limitation (BDRS subscales). Second, scalogram analysis shows that the items form a hierarchically ordered scale consistent with the course of functional loss established for dementing disease. Finally, prospective data indicate that the scale is sensitive to disease progression; dependence levels increased significantly over time.

Given the large number of existing scales for rating $\mathrm{AD}$ severity, a newly developed scale should not only be reliable and valid but should fill some unmet assessment need. The present data can only partially speak to this issue. This scale differs from functional assessment and caregiver burden measures in its focus on the needs of the elder and hence is perhaps most relevant as a clinical tool for predicting the service needs of the AD patient across the course of disease.

Insofar as the scale offers a more sensitive assessment of such service needs, the dependency scale may also serve as a research tool. While nursing home admission is typically used as an endpoint in the natural history of $\mathrm{AD}$, it is an imperfect measure, reflecting other factors in addition to the service needs of the patient. The dependency scale documents levels of service need directly, and in this way may offer a more sensitive endpoint for studies of $\mathrm{AD}$ progression. A key feature of the scale is that it is independent of the specific caregiving site and does not specify who is providing caregiving support.
We can demonstrate that the scale is not simply an alternate version of the Blessed Dementia Rating Scale (BDRS). While the items differ, we considered the possibility that the two scales simply tap aspects of a single functional domain. However, the dependence level and BDRS scores were both independently related to mMMS scores, suggesting that these scales diverge and tap some unique aspects of the disease presentation. Also, controlling for BDRS self-care deficits did not remove the significant association between dependency and the course of disease. Also, preliminary evidence suggests that dependency level may be a better predictor of living arrangement than the BDRS selfcare measure.

The scale needs refinement in a number of areas. Scoring for the more severe levels of dependency (levels 4 and 5) remains to be clarified. In particular, the relative position of the need for diapering (item $L$ ) and the need to be fed (item J) as indicators of severe dependency remains unclear. We expected the need to be fed to represent a less severe form of dependency than the need for diapering. However, scalogram analysis indicated that the need to be fed may be the more severe form of dependency. Other research with conmunity-dwelling elders has noted this ambiguity (18). Prospective analysis of the dependency experience of this cohort may help clarify this point. Aside from this exception, the scalogram ordering of dependency items is quite similar to the original scoring conventions for dependency level (i.e., while the order of the items varies somewhat, their grouping as levels is preserved).

Similarly, factor analysis of the dependency items shows only a slightly different picture of dependency levels. The order of severity of the dependency items is identical, but only three broad areas of dependency emerge. It should be noted, however, that the emergence of three factors in some ways supports the original scoring convention. The need for help with chores (item D), when combined with endorsement of any one of the first three items, gives rise to our intermediate second level of dependency (see Chart 2). As in the scalogram analysis, the position of "needs to be fed" (item $\mathrm{J}$ ) is again ambiguous.

A second area of refinement involves decisions to use item total scores, the dependency levels reported here, or the separate subscales that emerge in factor analyses. For clinical use, dependency levels may be most useful; and we have chosen to assess the scale according to such levels. However, for research purposes, it would seem most appropriate to examine each of these indices. Further research with this cohort and replication of these results using other samples will be valuable for determining optimal scoring of the scale. 
In conclusion, it would appear that dependency is a distinct, measurable component of dementing disease, which ought to be considered an important outcome in studies of AD that stress the service needs of patients. The dependency scale proposed here, along with the allied measure of equivalent institutional care, may be an important tool in clinical and epidemiologic research on $\mathrm{AD}$.

\section{ACKNOWLEDGMENTS}

This research was supported by grants AG-07370, AG-08702, and AG07232 from the National Institutes of Health, and by the Charles S. Robertson Gift for Alzheimer's Disease.

Address correspondence to Dr. Yaakov Stern, Gertrude H. Sergievsky Center, 630 West 168th Street, New York, NY 10032.

\section{REFERENCES}

1. Blessed G, Tomlinson BE, Roth M. The association between quantitative measures of dementia and of senile changes in the cerebral grey matter of elderly subjects. Br J Psychiatry 1968;225:797-811.

2. Stern Y, Mayeux R, Hesdorfer D, Sano M. Measurement and prediction of functional change in Alzheimer's disease. Neurology 1990;40: 8-14.

3. Wasson JH, Sox HC, Neff RK, Goldman L. Clinical prediction rules: applications and methodological standards. $\mathrm{N}$ Engl J Med 1985; 313:793-9.

4. Gurland B. Dependency in the elderly of New York City: policy and service implications of the US-UK Cross-national Geriatric Community Study. New York: Community Council of Greater New York, 1978.

5. Keller DM, Kovar MG, Jobe JB, Branch LG. Problems eliciting elders' reports of functional status. J Aging Health 1993;5:306-18.

6. Lawton MP, Brody EM. Assessment of old people: self-maintaining and instrumental activities of daily living. Gerontologist 1969;9:179-86.
7. Lawton MP, Kleban MH, Moss M, Rovine M, Glicksman A. Measuring caregiving appraisal. J Gerontol Psych Sci 1989;44:P61-P71.

8. Whitlatch CJ, Zarit SH, von Eye A. Efficacy of interventions with caregivers: a reanalysis. Gerontologist 1991;31:9-14.

9. Folstein MF, Folstein SE, McHugh PR. "Mini-Mental State": a practical method for grading the cognitive state of patients for the clinician. J Psychiatr Res 1975;12:189-98.

10. Stern Y, Sano M, Paulson J, Mayeux R. Modified Mini-Mental State Examination: validity and reliability. Neurology 37(Suppl 1);1987:179.

11. Hughes CP, Berg L, Daniziger WL, Cohen LA, Martin RL. A new clinical scale for the staging of dementia. Br J Psychol 1982; 140: $566-72$.

12. McKhann G, Drachman D, Folstein M, Katzman R, Price D, Stadlan EM. Clinical diagnosis of Alzheimer's disease: report of the NINCDSADRDA Work Group. Neurology 1984;34:939-44.

13. American Psychiatric Association. Diagnostic and statistical manual. 3rd ed., rev. Washington, DC: American Psychiatric Association, 1987.

14. Stern Y, Folstein M, Albert M, et al. Multi-Center Study of Predictors of Disease Course in Alzheimer's Discase (the "Predictors Study"): I. Study Design, Cohort Description and Intersite Comparisons. Alzheimer's Dis Related Disorders 1993;7(1):3-21.

15. Richards M, Folstein M, Albert M, et al, Multi-Center Study of Predictors of Disease Course in Alzheimer's Disease (the "Predictors Study"): II. Baseline Findings. Alzheimer's Dis Related Disorders, $1993 ; 7(1): 22-32$.

16. Guttman LL. The basis for scalogram analysis. In Maranell GM, ed. Scaling: a sourcebook for behavioral scientists. Chicago: Aldine, 1974.

17. Nie, NH, Hull CH, Jenkins JG, et al. Statistical package for the social sciences, 2nd ed. New York: McGraw Hill, 1975.

18. Travis SS, McAuley WJ. Simple counts of the number of basic ADL dependencies for long-term care research and practice. Health Serv Res $1990 ; 25: 349-60$.

Received June 15, 1993

Accepted October 13, 1993 